\title{
Advancements in sensor technology with innovative and significant research publications: how to write that perfect paper?
}

\author{
Antje J. Baeumner ${ }^{1} \cdot$ Hua Cui $^{2} \cdot$ Günter Gauglitz $^{3} \cdot$ Maria C. Moreno-Bondi $^{4} \cdot$ Sabine Szunerits ${ }^{5} \cdot$ Adam T. Woolley $^{6}$
}

Accepted: 18 May 2021 / Published online: 1 June 2021

(C) Springer-Verlag GmbH Germany, part of Springer Nature 2021

The general immediate reaction to the question posed may be that it should not be different from writing any other scientific paper, and in general terms, this is true for all research seeking to develop new technologies. Significant scientific advancements are based on innovation, which is the driving force for moving any research beyond the state-of-the-art. For sensor research specifically, innovation or novelty is the creation, development, and implementation of a new sensing concept, interface, and/or sensing ligand. Its aim is to improve selectivity or sensitivity or to offer other competitive advantages. Innovative sensor papers should thus describe a new technology that differs from previous ones by a large extent and addresses notable, existing hurdles or drawbacks, and hence an unresolved problem in the sensing field. Its advantages should be demonstrated by comparison of the performance to other analytical techniques or sensors. Analytical metrics for comparison include sensitivity, selectivity, dynamic range, limit of detection, and response time, and also parameters such as shelf-life, stability, and reproducibility. A valuable contribution to science is not demonstrated simply by the statement:

Published in the topical collection celebrating $A B C s$ 20th Anniversary.

All authors contributed equally.

Adam T. Woolley

woolley-abc-editor@byu.edu

Antje J. Baeumner

antje.baeumner@ur.de

Hua Cui

hcui@ustc.edu.cn

Günter Gauglitz

guenter.gauglitz@uni-tuebingen.de

Maria C. Moreno-Bondi

mcmbondi@ucm.es

Sabine Szunerits

sabine.szunerits@univ-lille.fr "this has never been done before." Many things have never been done before, but most of those also would not truly contribute to scientific knowledge, if they were to be carried out. Take the example of carbon quantum dot synthesis for enhanced biosensing: even though nobody has used yellow carrots in contrast to orange carrots for carbon dot synthesis, yellow carrot carbon dot synthesis alone is not sufficient to make an innovative sensor design, even though "it has never been done before to our knowledge."

In recent years, Analytical and Bioanalytical Chemistry has been focusing on the publication of sensor-related papers, including the criteria of novelty, analytical improvements and expanding the state-of-the-art. This editorial, written for the 20th anniversary of $\mathrm{ABC}$, takes the opportunity to highlight important guidance for writing excellent papers in this field of (bio)analytical chemistry. Our goal is for authors to improve their ability to present those relevant experiments and findings that are of greatest interest to the sensor community and the scientific community at-large, and hence participate best in the scientific discourse.
1 Institute for Analytical Chemistry, Bio- and Chemosensors, University Regensburg, Universitätsstraße 31, 93053 Regensburg, Germany

2 Department of Chemistry, University of Science and Technology of China, 96 Jinzhai Road, Hefei 230026, Anhui, China

3 Institute for Theoretical and Physical Chemistry, Eberhard-Karls-University, Tübingen, Germany

4 Department of Analytical Chemistry, Faculty of Chemistry, Complutense University, 28040 Madrid, Spain

5 Institut d'Electronique, de Microélectronique et de Nanotechnologie (IEMN, UMR CNRS 8520), Université de Lille, 59652 Villeneuve d'Ascq, France

6 Department of Chemistry and Biochemistry, Brigham Young University, Provo, UT 84602-5700, USA 
We start by refocusing on novelty and relevance within the constraints of this field of research, where ease-of-use and applicability to real-world scenarios are mandatory characteristics. Rendering a sensor more sensitive via the addition of countless assay steps does not necessarily advance the field further, since reduction of assay steps enables ease-of-use and reproducibility. However, it could be valuable in select circumstances to add assay steps, if those elucidate mechanisms or point toward future solutions to a major existing challenge. Actual applicability of sensors to real-life situations is most often a requirement. Thus, is a sensor that has never seen a real-world sample and has only been used with pure buffer solutions relevant? It still could be, for example, if the sensor entails a truly novel concept. Keep in mind, scientific sensor papers should ensure an increase in scientific knowledge and be highly relevant to your peers.

Where is such scientific knowledge urgently needed? The following illustrate a few key examples. (i) Biosensors are chemical sensors in which the recognition system utilizes a biochemical mechanism for the interaction process in/at the recognition layer. Bioassays, in contrast, may use the same mechanism but do not have a direct connection with a transducer. Importantly, trends and developments of this interaction found in both biosensors and bioassays have opened new perspectives in the sensing field and are therefore also of great interest to the sensor community. One example is the use of intracellular markers that generate an analytical signal by direct interaction of the sample with the recognition element in solution or in situ. In this bioassay, signals are monitored using a small imaging device rather than an in situ transducer. (ii) A myriad of nanomaterials (e.g., metal, metal oxide, carbon or polymer nanoparticles) have enabled a considerable enhancement of the sensitivity and selectivity for chemical and biochemical species detection. Often neglected is their applicability to real-world samples, their reproducible (and potentially large-scale) production, and head-to-head comparison to bulk materials. Information is hence needed about their analytical performance and the availability of reproducible calibration methods in light of stability, fouling, signal drift, target separation, and interference effects. (iii) Considering the singular importance of (bio)recognition elements in sensors, the development of new molecular (bio)recognition elements (aptamers, peptides, antibodies, polymers, etc.) is of great interest including studies on their selectivity, affinity, solubility, stability, and immobilization.

Understanding the analytical performance of a technique is the bread and butter of any good paper. As a sensor is a miniature analytical device for measuring the concentration of an analyte species, standard IUPAC protocols and definitions should be utilized, just as in any analytical publication. These include calibration characteristics (sensitivity, operational and linear concentration range, limit of detection (LOD), and/or limit of quantitation (LOQ)), selectivity, steady-state and transient response times, sample throughput, reproducibility/repeatability, stability, and lifetime (Orange Book, http://old.iupac.org/publications/analytical_ compendium/). Keep in mind that sensitivity and specificity have different meanings in analytical chemistry compared with biomedical assays and their use should be clearly described in the "Methods" section of a paper. While reversibility and short response time can be achieved for most chemical sensors with low equilibrium constants, biosensors exhibit high selectivity due to their large equilibrium constants, with the consequence of small dissociation rate constants. Therefore, most biosensors are not reversible, requiring high-quality regeneration procedures or excellent batch-to-batch reproducibility/repeatability to facilitate standard protocols that require blank and replica concentration measurements. Thus, biosensor papers in particular also need information on the quality of regeneration, effects of the shielding layer on top of the transducer, working range, and stability of immobilized recognition elements. As biosensors are based on biomolecular interaction processes, they exhibit a non-linear, sigmoidal calibration curve in the usual semi-logarithmic plot. In order to determine the LOD and LOQ, the 95\% confidence belt and associated minimum detectable concentration and reliable detection limit should be calculated for sigmoidal calibration curves [1]. A frequently updated resource from the US FDA offers further guidance on bioanalytical method validation (https://www.fda.gov/files/ drugs/published/Bioanalytical-Method-Validation-Guidancefor-Industry.pdf).

The comparability of the new sensor to existing methodology can be addressed on a variety of levels. Where available, reference materials or alternative reference methods should be used to validate a new sensor. As a minimum, an objective analytical performance comparison should be given to related papers or even commercial systems. Keep in mind that editors, reviewers, and $\mathrm{ABC}$ readers will easily spot a biased comparison that ignores relevant prior work; therefore, please provide an honest discussion of comparability. If you want to highlight the convenience, cost, simplicity, etc., of a sensor, plan to use quantitative metrics ( $€$, time, assay steps, etc.). These important details will make the sensor's advantages clear to the editor, referees, and future readers. It is important to keep in mind that a sensor may still be significant if it is not better than 
prior ones in every single metric; however, it should clearly improve over existing methodologies in important ways as discussed in the beginning of this editorial.

In the end, these guidelines for that "perfect sensor paper" offer reminders about things to be included that result in a high-quality manuscript and help avoid common roadblocks that could keep novel work from being published. Much of the general guidance given herein for sensor papers is also relevant for any paper submitted to $\mathrm{ABC}$ : be sure to state what is innovative and significant, and provide appropriate metrics for comparison. $\mathrm{ABC}$ is excited to consider and publish sensors papers that meet this guidance, along with other papers that provide major advances in the fields of analytical and bioanalytical chemistry.

\section{Reference}

1. Fernandez-Ramos MD, Cuadros-Rodriguez L, et al. An IUPACbased approach to estimate the detection limit in co-extractionbased optical sensors for anions with sigmoidal response calibration curves. Anal Bioanal Chem. 2011;401:2881-9.

Publisher's note Springer Nature remains neutral with regard to jurisdictional claims in published maps and institutional affiliations.

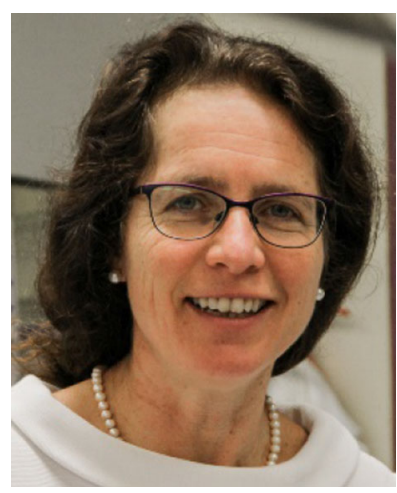

Antje J. Baeumner has been Director of the Institute of Analytical Chemistry, Chemoand Biosensors at the University of Regensburg (Germany) since 2013. Previously, she was Professor in the Dept. of Biological and Environmental Engineering at Cornell University, (Ithaca, NY, USA), where she still remains active as Adjunct Professor. Her research focuses on biosensors and miniaturized bioanalytical systems for clinical diagnostics, food safety,

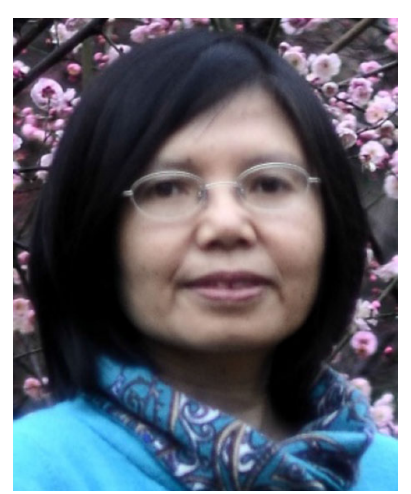

Hua Cui has been full Professor of Analytical Chemistry and Head of the Analytical Division, Department of Chemistry, University of Science and Technology of China since 2000. Her research interests are analytical chemiluminescence (CL) and electrochemiluminescence (ECL), and their applications in interdisciplinary fields including public health, food safety, and environmental monitoring.

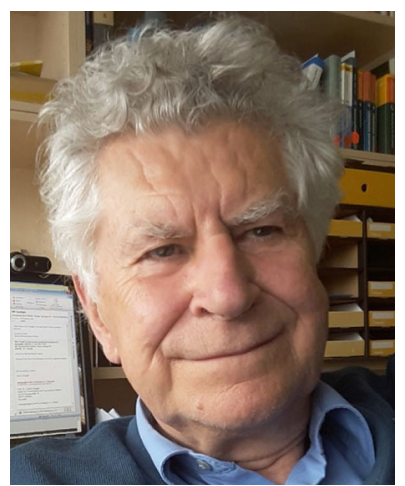

Günter Gauglitz is Senior Professor at the Eberhard Karls University of Tübingen working on analytical and physical chemistry. For more than 25 years, his main scientific interests have centered on research and development in chemical and biochemical sensors, with special focus on the characterization of interfaces of polymers and biomembrane surfaces, spectroscopic techniques, use of spectral interferometry to monitor changes in the optical thickness of thin layers, and the effects of Fresnel reflectivity at interfaces. He is one of the founding editors of Analytical and Bioanalytical Chemistry.

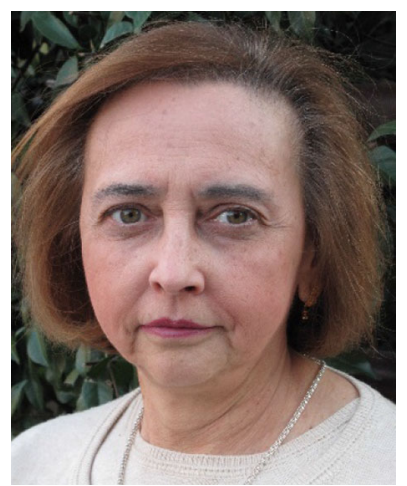

Maria C. Moreno-Bondi has been Professor of Analytical Chemistry at Complutense University of Madrid (UCM), Spain, since 2008. Her current research interests lie in the development of luminescent optical sensors and biosensors, molecularly imprinted polymers, nanomaterials, phage display techniques, epitope-mimicking peptides, recombinant antibodies, and their applications to food, clinical, and environmental analysis. 


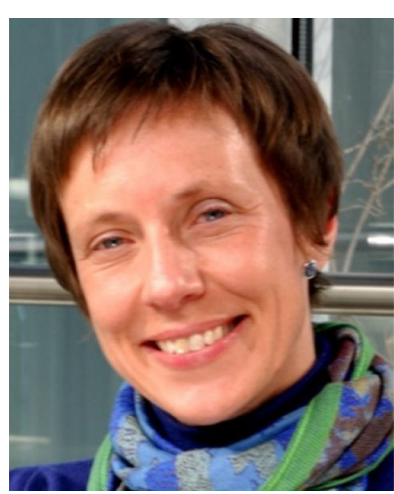

Sabine Szunerits obtained her $\mathrm{PhD}$ from Queen Mary and Westfield College, University of London, UK, and since 2010 has been Full Professor of Chemistry at University of Lille, France. Her research interests are in the area of materials science with emphasis on the development of novel nanostructures and approaches for nanomedical applications as well as sensing-related issues. In 2018, she was awarded the Médaille d'argent CNRS for her contribution to sensing and nanomedicine.

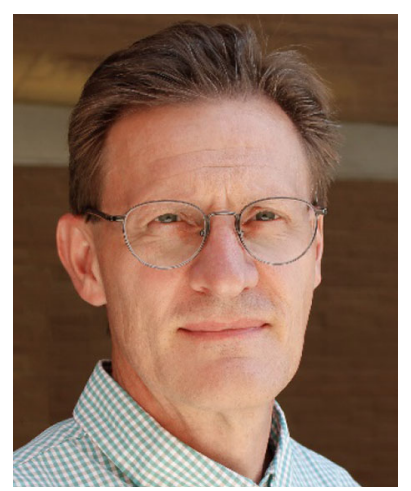

Adam T. Woolley is Chair Editor of Analytical and Bioanalytical Chemistry, and Dean of Graduate Studies and University Professor at Brigham Young University in Provo, UT, USA. His research focuses on 3D-printed integrated microfluidic devices for biomarker measurement, analytical systems for the identification of bacteria and antibiotic resistance genes in sepsis, and biotemplated fabrication of nanomaterials with electronics applications. 